\title{
Knowledge of breast cancer in women in Sierra Leone
}

\author{
JHEE Shepherd, Msc Nursing (MCH) \\ School of Nursing, University of KwaZulu-Natal \\ PA Mclnerney, Ph D \\ School of Nursing, University of KwaZulu-Natal
}

\section{Keywords:}

non-abusive intervention programme (NIP), breast cancer, Sierra Leone, knowledge

\section{Correspondence address:}

Prof PA McInerney

School of Nursing

Howard College Campus

University of KwaZulu-Natal

PO Dalbridge

4041

Tel : (031) 260-2497

Fax : (031) 260-1543

E-mail :mcinerneyp@ukzn.ac.za

\section{Abstract: Curationis 29(3): 70-77}

Breast cancer has been described as one of the life-threatening diseases affecting women and is a major problem in women's health issues. The unrecorded number of cases of breast lumps and breast cancer observed in women in Sierra Leone prompted the researcher to organize a "Breast Week" during which 1200 women were educated on breast cancer and the importance of breast health. This research is a follow up of the "Breast Week" which was organized in Freetown, Sierra Leone The specific objective of this study was to assess whether the knowledge and teachings given to the women who participated in this project was fully understood. A sample size of 120 women $(10 \%)$ who participated in the "Breast Week" was obtained through systematic sampling.

A quantitative approach was adopted and a structured interview schedule guided the data collection process. The data were processed through use of SPSS and Microsoft Excel. Texts from open ended questions were categorized and frequency counts were applied to the data. It was found that the majority $(96.6 \%)$ of the women had some knowledge of breast cancer. They linked breast cancer to the signs and symptoms associated with it and were able to describe the disease as one that kills women if not promptly detected and/or treated appropriately. Findings indicate that the majority of the women are aware of the dangers of the disease and had knowledge of someone who had died of breast cancer $(59.2 \%)$. An assessment of the effectiveness of knowledge on breast cancer showed that these women could identify breast cancer as a disease that affects women and may cause death if not detected on time.

\section{Introduction and problem statement}

Cancer was previously known to be a disease of the affluent world, but in recent years the incidence of cancer in developing countries has soared (WHO, 1997:2). According to the World Health Organization (Smyke, 1993:19) cancer is now also a third world problem, and if existing trends continue, cancer mortality is expected to rise in the future in nearly all regions of the world (Smyke, 1993:19). Breast cancer is said to be the leading cause of cancer among women in most developed countries and in many developing countries (Smyke, 1993:95).
In South Africa, the Cancer Association of South Africa (CANSA, 1993:1) estimated that cancer affects over 4,000 women per year and that the most common cancers are breast and cervical cancer. According to the American Cancer Society, about 1 in every 8 women in the US is likely to be affected by breast cancer. Every year, $40 \%$ of these women die from the disease. "There has been an increase of $22 \%$ in breast cancer mortality over the past two decades" (Smyke, 1993:95). A quarter of breast cancers are diagnosed before the age of fifty, and the survival rate is five years after detection of the disease (CANSA, 1993:1).

Breast cancer is and still remains one of 
the cancers affecting all age groups of women worldwide (WHO, 1997:3). "Treatment is less successful for breast cancer than for cervical cancer" (Smyke, 1993:95). There are different patterns in the incidence of breast cancer between women in industrialized countries and those in the developing countries (Smyke, 1993:19). In the industrialized countries, lung cancer is said to be replacing breast cancer as the most common cause of death due to cancer among women (Smyke, 1993:95). Smyke further notes that, for the most part, the differences reflect differing lifestyles but that these patterns change rapidly (Smyke, 1993:25) and that one-third of cancers could be prevented if women are armed with the right knowledge. Current trends in women's health globally, are revealing the high incidence of breast cancer in women (Smyke, 1993:95).

Despite recognition of this fact, a country like Sierra Leone has no breast cancer policy in place to address this threat. Presently, there is no institute undertaking research in cancer in the country and no screening facilities, e.g. a mammogram, available in the whole country. The researcher had noticed that several women, young as well as old, nurses as well as laypersons, had had recent surgery to remove breast lumps or had died of breast cancer. These cases have gone unresearched except for the case histories as told by the survivors themselves. Furthermore, studies have not been conducted to determine the severity of this problem in Sierra Leone.

Sierra Leone as a country is faced with the problem of accessing reliable data. This situation has been worsened by the 10-year rebel war in the country when a number of health facilities were destroyed and rendered non-functional (UNICEF, 1999: 145). Women do not have access to vital information regarding their health. Basically, women rely on radio discussions or health talks during visits to the antenatal clinic for information on reproductive health.

Emphasis on early detection is seen as vital especially in developing countries where there is no access to modern technology to detect breast cancer. The lack of basic knowledge and an effective information delivery system for breast cancer further threatens the life and well being of women. Breast cancer is silently killing women, many of whom have no knowledge and continue to be ignorant about breast cancer and screening methods for early detection. Findings from this study will serve as an initial stepping stone and starting point to meet the growing challenges of breast cancer that are currently going unnoticed in Sierra Leone.

\section{Setting}

The study was conducted in Sierra Leone, a country located on the west coast of Africa between Guinea and Liberia. It has an area of about 72,800 square kilometres. Freetown is the capital city of Sierra Leone and is located in the western part of the country. In 2000 , Sierra Leone had a population of a little over 5 million, $51 \%$ of whom were women. Sierra Leone is rated as one of the least developed countries with the lowest quality of life and women are at a disadvantage in terms of literacy (UNICEF, 1999:67). The country was plagued by a ten-year rebel war which has further compounded the problem of women gaining access to quality care and information on women's health issues.

During clinical practice the researcher had observed that there appeared to be more women admitted to the hospital with breast cancer. Some of the women had sought help from traditional healers and had commenced traditional healing methods such as applying herbs to their breast. This resulted in inflammation of the breast and it was at this stage that they reported to the hospital seeking medical help. It was not known whether these women recognized the serious nature of breast cancer or knew the signs and symptoms of the disease. It was against this background that the researcher organized a "Breast Week", as part of health promotion activities, to educate women on breast cancer.

A radio discussion on breast cancer and methods of detection was held in Freetown prior to the breast week. The 'Breast Week' was advertised on the radio programmes and in the communities by nurse-midwife tutors and nurse-midwives in training. Over 1200 women participated in the breast week and had their names and contact details entered in a registration book. These women received health education in order to create an awareness of breast cancer, methods of early detection and the importance of early reporting of abnormalities of the breast to the hospital. They were informed that they could be contacted by the researcher in future for future studies to which they all agreed. This study was therefore a follow up measure by the researcher to ascertain whether the information provided during the breast week on breast cancer was understood by the women. An assessment of the effectiveness of the proceedings during the breast week was the prime focus of this study.

\section{Purpose and objectives of the study}

The purpose of this study was to evaluate the knowledge of breast cancer in women in Freetown and its impact towards reinforcing positive learning outcomes. The main focus of the study was breast cancer as one of the diseases affecting women. The objectives of this research were to:

- $\quad$ To assess if the information given to the women regarding breast cancer during the breast week was fully understood.

- To assess if the information given during the breast week had created an awareness on the serious nature of breast cancer.

- $\quad$ To identify gaps, weaknesses and strengths in the knowledge imparted during the breast week.

\section{Significance of the study}

The information generated from this study will contribute to improving awareness on the level of knowledge of women on matters regarding breast cancer. Findings will be utilised in formulating teaching plans to address issues such as breast health among women at all levels in the Sierra Leone community. Therefore gaps and deficiencies highlighted in the study could contribute to health service planning.

\section{Validity and reliability}

A pilot study was conducted before the actual study started as a means of determining the reliability of the instrument. This involved testing the actual instruments on a small sample taken from the sample population under study (Polit \& Hungler, 1997:44). The pilot study was conducted a week before the 
study was carried out. Five women were selected from the target population. These women were not included in the main study. The contents of the structured interview schedule administered to the women were limited to only health teachings given during the breast week.

\section{Ethical considerations}

Permission to undertake this study was sought from the University's Research Ethic Committee; the Ethics Committee on Health, Freetown, Sierra Leone; the Consultant in-charge of the main referral hospital and the participants. Informed consent was obtained from the participants. Anonymity and confidentiality were ensured. Participation in the research was voluntary. Participants were offered the opportunity to withdraw from the study at any time and were assured of no ill effects. Participants were given a pamphlet on breast self-examination (BSE) and a pink ribbon as a token of appreciation for participating in the study.

\section{Literature review}

According to Fentiman (1999:42), breast cancer is the most prevalent type of cancer in women. Similarly, CANSA (2000:1) reports that breast cancer is one of the most common cancers among South African women affecting over 5000 women per year. Breast cancer is said to be the most prevalent cancer amongst White females and the second most common cancer amongst Black women.

\section{Incidence}

According to CANSA (2004:1), one out of ten lumps in the breast is cancerous. In South Africa in 1988, 3324 per 100,000 women were diagnosed with breast cancer. The age group most commonly affected was between the ages of 55 and 85 years, with the highest incidence amongst the $80-84$ year age group (South African National Cancer Registry, 2001:1). The American Cancer Society estimated that approximately 130,900 new cases of malignant breast tumours were discovered in 1987, and approximately 41,300 women died of the disease (American Cancer Society, 2003:1). In the United States, the overall incidence of breast cancer increased in the early 1970 's to the early 1990's and then decreased approximately $0.6 \%$ from 1991 (American Cancer Society, 2003:1). This drop has been linked to advances in medical research and earlier detection of the disease. Consequently, the overall survival rates have increased.

\section{Risk factors}

Breast cancer is an issue of concern for every woman in today's society. According to the National Cancer Institute (2002:1) being female is the highest risk factor for breast cancer, one out of eight women in the United States has a lifetime risk of developing breast cancer. Increasing age is discussed as the second highest risk factor (American Cancer Society, 2003:2). Breast cancer is said to be common in White women across all age groups and is more frequent in black women under the age of 45 (Keitel \& Kopala, 2000:13). Seventy five percent of new cases of breast cancer are said to occur in women age 50 and older. However, Keitel \& Kopala (2000:13) have stressed that younger women tend to experience a higher mortality from the disease.

The Cancer Association of South Africa (2004:1) included in their list, risk factors such as obesity, early onset of menses, late menopause, prolonged use of hormone replacement therapy with high dosage, and a high fat diet. In addition to the above mentioned, Keitel \& Kopala (2000:15) list age, genetics, hormonal activity, lifestyle factors such as diet, and excessive alcohol use as contributory risk factors. Personal or family history of breast cancer and environmental factors are also said to be risk factors for breast cancer (Dixon, 1995: 19). However, Keitel \& Kopala (2000:15) state that although a family history increases one's risk, 70\% of women diagnosed with breast cancer had no known risk factor. It was further disclosed that women who once had breast cancer are at increased risk for a new primary breast tumour. Smoking was also identified as a risk factor for developing breast cancer and research findings have established that smokers have a $25 \%$ greater risk of dying from breast cancer than non-smokers (Keitel \& Kopala, 2000:16). In addition to these factors, Keitel \& Kopala (2000:16) cited stress and depression as being linked with breast cancer. They however concluded that this theory has not yet been proven by research.

\section{Detection}

Detection of cancer at an earlier stage is linked to better outcomes (Haskell,
1995:10). An article by the Cancer Association of South Africa (http:// www.cansa.co.za) states that "Breast cancer can be cured if caught on time". The need for one to be knowledgeable about their breasts has been discussed by Northrup (1999: 313). Similarly finding out about the status of the breasts is being promoted by Breast Cancer Associations worldwide as no one is immune to breast cancer (National Cancer Institute, 2000:1). In a pamphlet on breast cancer, the Cancer Association of South Africa (2000:1) stressed that regular examination can set women free and that early detection is the key to survival. In order for a woman to detect breast cancer early she needs to be aware of the changes that are suggestive of breast cancer.

This intervention involves performing monthly breast self-examination, observing for and noting abnormal changes in the breasts and reporting to the doctor promptly. An annual physical check up by the doctor is also recommended. Women are also advised to go for a mammogram especially if they happen to be in the high-risk group (Berger \& Bostwick, 1994:22). Another new development in breast cancer detection is that of using heat sensitive pads (American Family Physician, 2003:2). These pads are worn inside the brassiere and act as an early indicator of breast cancer. Currently it is approved as a supplement to the more traditional tests (American Family Physician, 2003:2).

\section{Diagnosis}

Watts (1990:4) maintains that the exact mechanism by which a cancer arises is complex and poorly understood and the growth pattern and metastatic potential of a cancer is usually predetermined in advance before clinical detection of the cancer. In order to make a proper diagnosis, Keitel \& Kopala (2000:20) point out that it is imperative for a woman to be referred to a breast surgeon for consultation if a solid mass or abnormal change is detected. However, there are some observed abnormal changes or breast lumps that are not cancerous. One of the traditional methods of breast cancer screening includes clinical breast examination (CBE). LoBuono (2001:1) describes $\mathrm{CBE}$ as being an integral part of a complete breast cancer-screening program in which a physician palpates the breasts, looking for subtle changes in the breast tissue. Diagnosis of breast 
cancer is only made after a thorough examination by the doctor followed by the necessary investigations. Biopsies are generally performed to determine whether a lump is cancerous (Huffman, 2000:1). Watts (1990:134), however maintains that with the use of breast screening procedures some cancers can be detected. The mammogram is an $\mathrm{x}$-ray examination of the breast tissue done by a radiologist in a breast diagnostic centre or at the physician's office. It is used as a screening method for breast cancer as well as in its diagnosis (Berger \& Bostwick, 1994:20). An expert radiologist may be able to diagnose some benign conditions.

\section{Treatment and effects}

Treatment of breast cancer is dependent on the stage and type of cancer as well as the age and health of the woman (Haskell, 1995:21). Literature also supports the fact that early detection and diagnosis are the best treatment of breast cancer (Berger \& Bostwick, 1994:12). Basically, the treatment of breast cancer involves surgery in which the lump or the whole breast is removed in addition to the surrounding lymph nodes (Haskell, 1995:18). This management is performed in conjunction with radiotherapy. In the late stages, or to prevent reoccurrence, chemotherapy is used alongside surgery and radiotherapy (Dixon, 1995:43-44). Endocrine therapy and vaccines are also part of the treatment offered to women who are likely to develop cancer or are affected with cancer (Haskell, 1995:21). Haskell (1995:22) however noted that the treatment of each patient should be individualized. Breast cancer and its treatment have physical and psychological implications for women.

Physical and psychological trauma are associated with breast surgery (Keitel \& Kopala, 2000). If treatment of breast cancer involves mastectomy, a woman is faced not only with the prospect of a frightening disease, but also with the loss of a part of her body which is essential to her feminity (Keitel \& Kopala, 2000:28). Women who are concerned about their body image after removal of the affected breast often opt for reconstruction surgery. This involves tissue replacement and plastic surgery. Advancement in technology has also made it possible for women to wear prostheses under their clothes to make the breasts appear normal.

\section{Prognosis}

The prognosis of breast cancer is influenced by a myriad of factors some of which cannot be prevented (Haskell, 1995:10). So far there is no specific intervention to stop cancer, but according to researchers if cancer is detected in the early stages the prognosis is good (Keitel \& Kopala, 2000; Haskell, 1995:10). The chances of a complete recovery from breast cancer are highest $(90 \%+)$ when the disease is detected early (National Cancer Institute, 2000:2). This means that the percentage of patients surviving five years or longer after diagnosis of breast cancer, apparently detected the cancer at its early stage. If this is the case, then women need to be more knowledgeable about their body's function in order to note abnormal changes. The overall impression is that the prognosis of breast cancer depends not only on a single factor but on several of them.

\section{Theoretical framework}

Orem's Self-Care Nursing Model (1980) was selected for this study, as it addresses the key concepts of this research. Lack of knowledge of breast cancer and methods of detection spell out the "Self-Care Deficit" according to Orem's perspective. Provision of knowledge and skills is required if the care abilities are less than those required for meeting a known self-care demand. Information is power and helps bring about a change in attitude. An environment that promotes personal development in relation to becoming able to meet those needs is required (Orem, 1980:52).

\section{Research Design}

A quantitative, descriptive-exploratory research design was used to assess the knowledge of breast cancer in women in Sierra Leone. A structured interview schedule consisting of two sections was administered to the respondents as a means of collecting data in order to arrive at responses that could be quantified and analyzed as numeric values. The first section covered the socio-demographic information of the respondents, whilst the second section covered the theoretical knowledge base of breast cancer.

\section{Population and Sample}

The target population for this study was those women who attended the 'Breast
Week'. A total number of over 1200 women participated in the 'Breast Week' held in November 2002 in Freetown, Sierra Leone. Every tenth woman on the registration list of women who took part in the 'Breast Week' was systematically selected as a participant for this study, giving a total of 120 women.

\section{Data Collection}

The data were collected over a period of four weeks. The participants for this study were contacted through a list containing their names and contact addresses. Their permission to participate in the study was sought verbally and informed consent was obtained. Based on this, a structured interview schedule containing coded items in the form of closed and open-ended questions was administered to the 120 women. The first section of the interview schedule focused on socio-demographic data relevant to the study. The second section of the interview schedule contained questions focusing on the knowledge base of breast cancer, its causes, treatment and early detection methods.

\section{Data Analysis}

Data were analyzed using SPSS 11.5 and Microsoft Excel. Open-ended questions were analysed by grouping, in order of similarity the views expressed. Appropriate coding was allocated to the various groupings. Statistical tests were applied to variables such as age and level of education. The data did not lend itself to tests of statistical significance.

\section{Results}

\section{Socio-Demographic Data}

One hundred and twenty women participated in the study. Their ages ranged between 18 and 50 years and above. Half of the women fell within the age group 31-50 years. The majority of the women, $(77.5 \%)$, were married. Only $9.2 \%$ of the women were single and 5.8 $\%$ were widows. The majority of the respondents, $(70.8 \%)$, were traders who engaged in small and big businesses, 6.7 $\%$, were students, $50.8 \%$ had had no formal schooling, $34.2 \%$ had junior and secondary level education, with $11.7 \%$ having completed senior secondary schooling. None of the respondents had post school education. The total number of respondents who had received some form of schooling (59\%) was almost the same as those who had never been to school $(61 \%)$. A very large percentage of 


\begin{tabular}{|l|c|c|}
\hline What do you know about Breast Cancer? & $\begin{array}{c}\text { Frequency of } \\
\text { Responses }\end{array}$ & Percentage of cases \\
\hline $\begin{array}{l}\text { 1. It is a bad disease that affects the breasts, it kills women if not detected } \\
\text { early or treated on time }\end{array}$ & 70 & 58.8 \\
\hline $\begin{array}{l}\text { 2. It is recurrent lumps, boils, ulcers or sores on the breasts that refuse to } \\
\text { heal. A lump later resulting to sore in the breasts }\end{array}$ & 50 & 42.0 \\
\hline $\begin{array}{l}\text { 3. It is abnormal growth on the breasts, enlargement of the breast causing } \\
\text { swelling, itching, pain, skin changes and tenderness }\end{array}$ & 30 & 15.0 \\
\hline $\begin{array}{l}\text { 4. Hardening of the breast, abscess, or pus or bloody discharge from the } \\
\text { nipples }\end{array}$ & 12 & 10.0 \\
\hline $\begin{array}{l}\text { 4. Surgery is done on the breasts and the lump or affected breast is } \\
\text { removed, dangerous disease has no cure }\end{array}$ & 8 & 6.7 \\
\hline 5. No idea & 4 & 3.4 \\
\hline
\end{tabular}

NB. The numbers represented in categories 2 - 4 in Table 1 were not necessarily part of the original 70 women in category 1 . The frequency of responses in the column above represents multiple answers given by the respondents consequently adding up to the total percentage of cases in column 3.

the respondents, $(75.8 \%)$, were Moslems and only $24.2 \%$ belonged to the Christian faiths.

\section{Social Habits and Life Style}

With regards to lifestyle, the majority $(84.2 \%)$ reported that they did not drink alcohol. Those who drank alcohol (15.8 $\%$ ) differed in the type and amount of alcohol consumed per week. An even greater number, $(92.5 \%)$, responded that they did not smoke cigarettes. Over onethird $(38.3 \%)$ chewed kola nuts and did so in varying amounts. Kola nuts are found in countries such as Sierra Leone, Liberia, Guinea, and Gambia and are renowned for their addictive properties. It is a habit forming nut with a high caffeine content and is used as a pastime by some people in Sierra Leone. Kola nuts are used in making certain drinks such as coke, dye making and in traditional rites such as engagements or funeral ceremonies by certain tribes.

\section{Reproductive Health History}

Age at onset of menarche has been used as one of the risk indicators for breast cancer (Berger \& Bostwick, 1994:291). The greater percentage of the women $(85$ $\%$ ) reported having their first monthly period between the ages $12-15$ years, 9.2 $\%$ reported starting after the age of 15 years and $5.8 \%$ reported experiencing their first menstrual period at age 9-11 years. Almost all (97\%) of the respondents had children. Those who had up to three children constituted 36.7 $\%$ of the sample and those having delivered 4-7 children amounted to 47.5 $\%$. A small group of respondents (15.8 $\%$ ) had delivered eight or more children. Almost two-thirds $(64.2 \%)$ of the respondents had had their babies whilst aged 18-25 years. The analysis further showed that $24.2 \%$ of the respondents had their babies at age 18 and below. Only $3.3 \%$ of the respondents had their first babies at age 34 years and above and another $3 \%$ had no children.

With regards to breast feeding, all those who had had babies, breast fed all of their children giving a $100 \%$ breast feeding response. The majority $(69 \%)$, breastfed their babies for 1-2 years. Another $31 \%$ breast fed for more than 2 years. In terms of contraceptive use, just under twothirds of the respondents, $(63.3 \%)$, indicated that they had never used contraceptives, whilst $36.7 \%$ had used contraceptives. Of the 26 respondents currently using a contraceptive, 14 were using an injectable contraceptive. Only five reported using oral contraceptives and another five had had an IUCD inserted.

\section{Knowledge of breast cancer}

In response to the open-ended question "What do you know about breast cancer"? A number of varied answers and responses were given. These responses were summarized and categories are listed in Table 1. Just over half $(58.8 \%)$ described breast cancer as a disease that affects the breasts and kills women if not treated or reported early. Just under half $(42.0 \%)$ listed some of the signs and symptoms associated with breast cancer. Similarly, abnormal growth, enlargement, and skin changes were cited as indicators of breast cancer by $10 \%$ of the respondents. Abnormalities such as nipple discharges and hardening of the breasts were also cited by $10 \%$ of the respondents. A small percentage of respondents $(3.4 \%)$ indicated that they did not know what breast cancer was.

\section{Causes of Breast Cancer}

This was an open ended question, asking "What causes breast cancer"? The breakdown of varied responses on the causes of breast cancer is displayed in Table 2. Nearly three-quarters $(71.7 \%)$ of the respondents linked putting coins and other metals into one's brassiere as one of the causes of breast cancer. A small number, $(10.8 \%)$ had no knowledge of 
Table 2: Responses/Frequency on Causes of Breast Cancer $(N=120)$

\begin{tabular}{|l|c|c|}
\hline RESPONSES & FREQUENCY & (\%) \\
\hline $\begin{array}{l}\text { Traditional Beliefs/Practices such as putting coins or Metal in one's } \\
\text { Brassiere }\end{array}$ & 86 & 71.7 \\
\hline Bad Practices Around Breast Feeding/ Trauma to the Breasts & 15 & 12.5 \\
\hline No Idea & 13 & 10.8 \\
\hline Unknown Causes & 6 & 5 \\
\hline Total & 120 & 100.0 \\
\hline
\end{tabular}

what causes breast cancer. Only $5 \%$ of the respondents stated that the causes of breast cancer are unknown.

\section{Screening Method used for Early Detection of Breast Cancer}

Over half of the respondents, $(58.4 \%)$, considered Breast Self-Examination as one of the screening methods used in detecting breast cancer at its earliest stage. One-third $(33.3 \%)$, felt that breast cancer can only be detected by professionals, who included doctors and nurses, described by them as experts. They indicated that on their own they would not be able to detect breast cancer. A small proportion, $(8.3 \%)$, were however unable to give examples of screening methods through which breast cancer can be detected.

\section{Knew Someone Who Had Suffered From Breast Lumps or Cancer}

Over a half $(59.2 \%)$ said that they knew someone who had had a breast lump or who had suffered from breast cancer, whilst $40.8 \%$ of the respondents had no knowledge of someone who had suffered from or who had had a breast lump or cancer. Of those who knew someone who had breast lumps or breast cancer, 35.2 $\%$ said that these women had had the lumps removed. Over half of these women, $(64.8 \%)$, said that the person who they knew was suffering from breast cancer had died either before or after surgery.

\section{Discussion}

This study showed that $58.8 \%$ of the women could describe breast cancer as a life-threatening disease affecting women. There was however lack of knowledge of breast cancer expressed by some of the women (3.4\%) despite the educational activities during the breast week. One possible explanation could be that almost half of the sample $(61 \%)$ did not obtain formal schooling. This is consistent with the statistics of the country which reflect a low level of educational attainment and a high level of illiteracy among the women (UNICEF, 1999:69). A high participation of the women involved in trading was evident in the analysis. A possible explanation for this is that the hospital is located within walking distance of the market.

The literature infers that alcohol, cigarette smoking, a high caffeine intake, and an early menarche are relevant risk factors in breast cancer (Northrup, 1999:337). The results of this study showed that smoking was not a common habit among the women. Similarly, alcohol was consumed by only 10 women. Targeted interventions aiming at a risk-reduction in breast cancer could highlight the benefits of healthy lifestyle patterns in women who do not usually associate them with health benefits. A breastfeeding pattern has been associated with a risk reduction in breast cancer especially if continued for 18-24 months. According to the literature, women face greater risk of developing breast cancer if they have small families and do little or no breast feeding (The National Cancer Association of South Africa, 2000:49) and also if the age of giving birth to the first child is over 30 years (Berger \& Bostwick, 1994:291). Findings in the study showed that most of the women had large families and those who had had babies, breastfed all of their children for relatively long periods. The majority of the respondents, (63.9\%), breastfed their babies for 1-2 years.
Findings in this study also supports the fact that African mothers are known to practice breast feeding for longer periods some even up to two years (UNICEF, 1999:24). It can be argued then that women in African countries are at an advantage over countries where women do not breastfeed.

The need for comparative studies of the lifestyles of women in developed countries and developing countries and associated risks of breast cancer is of relevance. It is apparent from the study that these women practiced breastfeeding in line with cultural expectations and did not attribute it to a health practice associated with risk reduction of breast cancer. The need for nurses to identify and reinforce these positive health habits during health education and health promotion is important.

The findings suggest that the majority of the women had a good knowledge of what breast cancer is, as they ably described the disease in terms of signs and symptoms associated with the disease. The women could name abnormalities of the breast and linked the disease to the signs and symptoms associated with it. They recognized signs such skin changes and hardening of the breast as abnormal. This indicated that the women were aware of the serious nature of the disease. There was however a small number who mentioned that they did not know what breast cancer is. The development of an effective information system to constantly sensitize women on issues regarding their health is paramount especially for women who have no other means of accessing information. Considering the fact that nearly half of the respondents had no 
formal schooling, it is possible that they felt that they could not accurately describe the disease based on their level of understanding of the disease. Radio talks and mass media campaigns can be used to target women at grass roots level to continue to raise awareness on matters regarding their breast health.

In this study, the women (5\%) who stated that the cause is unknown appeared to be the most knowledgeable. This statement is supported by the literature as medical experts also state that the exact causes of breast cancer remain unknown (Huffman. 2000:2; Watts, 1990:4). Almost $72 \%$ of the women cited putting of coins or metal in their brassiere as a possible cause of breast cancer. On observation, the researcher sees this belief as one that is commonly shared by most women in the traditional communities in Sierra Leone. Keitel \& Kopala (2000:16) state that beliefs such as not wearing a brassiere or wearing an ill fitting brassiere, overactive or aggressive fondling of the breasts are held by women as being responsible for causing breast cancer. However, Keitel \& Kopala (2000:18) state that there is no evidence to support these beliefs. Nonetheless, women continue to believe that these events or conditions can cause cancer. Further research needs to be done to explore further traditional beliefs and myths and how they affect women's health. Again the role of nurses in research and the mass media in dispelling these myths is critical. The reality of the number of women dying from breast cancer was evident in the study as $59.2 \%$ of the women said that they knew of someone who suffered from breast cancer. Almost two-thirds of the women stated that the women known to them had died before or after surgery. It was not clear however whether these women reported the disease at a later stage. These findings have far reaching implications for the development of reproductive health policies on breast cancer screening for women in Sierra Leone.

\section{Recommendations}

\section{- $\quad$ Nursing Practice}

The issue of breast cancer is relevant in nursing and deserves special attention. The role of a nurse as a teacher, counselor and educator is crucial in women's health and cannot be underestimated in the health education of clients as discussed in Orem's Self-Care Model. This study demonstrated the beliefs and myths associated with the causes of breast cancer. This finding suggests that more education and means of disseminating information on breast cancer is needed. Positive habits such as breast feeding habits should be further reinforced through health education and the benefits promoted among women.

\section{- $\quad$ Nursing Education}

Nursing and midwifery curricula must include information on breast cancer and methods of screening for the disease. Breast cancer should be included as an important topic in women's health programs for nurses as a way of empowering women with knowledge on breast health.

\section{Conclusion}

Findings in this study highlight the issues surrounding women's knowledge of breast cancer in Sierra Leone. Given the observations of the researcher which motivated the "Breast Week" and the number of women in the study who knew of women who had breast cancer, there is the need for empirical studies to be conducted at national level.

\section{References}

AMERICAN CANCER SOCIETY 2003: Cancer facts and figures, 2001-2002. Atlanta, G. A: American Cancer Society. Retrieved May 23, 2003, at http:// www.cancer.org.

AMERICAN FAMILY PHYSICIAN, 2003: Screening for Breast Cancer: Recommendations and Rationale. Retrieved March 15, 2003, at http:// www.findarticles.com/cf_0/m3225/12.

BERGER, KJ \& BOSTWICK, J 1994: A Woman's decision. Breast care, treatment and reconstruction. $2^{\text {nd }}$ Edition. St Louis: Quality Medical Publishing Inc.

\section{CANCER ASSOCIATION OF SOUTH}

AFRICA, 1993: Breast cancer, facts and myths. (CANSA). Retrieved March 17, 2003 at http://www.cansa.co.za/facts

CANCERASSOCIATION OF SOUTH AFRICA 2004: Breast cancer. (CANSA). Retrieved March 17, 2003 at http:// www.cansa.co.za/facts

CANCERASSOCIATION OF SOUTH AFRICA 2000: A Pamphlet: Regular ex- amination can set you free of worry. Cancer Association of South Africa, Johannesburg.

CANCER ASSOCIATION OF SOUTH AFRICA 2001: Your guide to breast health. Cancer Association of South Africa, Rondebosch.

CANCER ASSOCIATION OF SOUTH AFRICA 2004: Research: Breast cancer. Retrieved June 24, 2003 at http:// www.cansa.org.za/Research/ facts_myths_breast.asp

DIXON, JM 1995: ABC of breast diseases. London: BMJ Publishing Group.

FENTIMAN, IS 1999: Challenges in breast cancer. London: Blackwell Series, Inc.

HASKELL, CM 1995: Cancer Treatment. $4^{\text {th }}$ Edition. Philadelphia: W. B. Saunders Company.

HUFFMAN, GB 2000: Should Family Physician Do Screening Breast Exams? American Academy of Family Physician. Retrieved March 15, 2003 from the World Wide Web: http://www.findarticles.com

KEITEL, MA \& KOPALA, M 2000: Counselling women with breast cancer. A guide for professionals. London: Sage Publications, Inc.

LOBUONO, C 2001: How to perform an effective clinical breast exam. Women's Health. Retrieved March 15, 2003 at http:/ /www.findarticles.com/cf

NATIONAL CANCER INSTITUTE 2000: What you need to know about breast cancer: An overview. U.S. National Institute of Health. Retrieved April 12, 2003 at www.cancer.gov

NATIONAL CANCER INSTITUTE (AMERICA) 2002: Cancer facts: Life time probability of breast cancer in American women. U.S: National Cancer Institute retrieved from World Wide Web http://cis.nci.nih.gov

NORTHRUP, C 1999: Women's bodies, Women's wisdom. The complete guide to women's health and wellbeing. London: The Bath Press.

OREM, DE 1980: Nursing Concepts of Practices. $2^{\text {nd }}$ Edition. New York: Mc Graw Hill Company. 
POLIT, DF \& HUNGLER, BP 1997:

Essentials of nursing research: Methods, Appraisal, and Utilization. $4^{\text {th }}$ Edition. Philadelphia: J.B Lippincott.

SMYKE, P 1993: Women and Health. World Development series. New Jersey: Zed Books Ltd.

SOUTH AFRICAN NATIONAL CANCER REGISTRY 2001: Cancer Registry epidemiology and early detection. Tygerberg: South African Cancer Association.

THE NATIONAL CANCER ASSOCIATION OF SOUTH AFRICA 2000: Cancer Research Report. NCA, Johannesburg.

UNICEF 1999: Situational Analysis of Women and Children in Sierra Leone. Freetown, Sierra Leone: UNICEF.

WATTS, CF 1990: Malpractice defense: Breast Cancer. New Jersey: Medical Economics Company Inc.

\section{WORLD HEALTH ORGANIZATION} (WHO) 1997: Cancer incidence in five continents. Vol. Vii. Lyon International Agency for Research on Cancer. Geneva: WHO. 